\title{
Mental Health Conditions are Associated With Increased Health Care Utilization Among Urban Family Medicine Patients
}

\author{
Colleen T. Fogarty, MD, MSc, Sapna Sharma, MBChB, MPH, Veerappa K. Chetty, PhD, \\ and Larry Culpepper, $M D, M P H$
}

Purpose: To assess the relationship between the presence of a mental health condition and health care utilization among family medicine patients.

Metbods: We used the Patient Health Questionnaire plus a posttraumatic stress disorder screen to measure 6 common mental health conditions. In a sample of 367 patients recruited from 3 urban family medicine practices affiliated with Boston University Medical Center, we measured self-reported health care utilization of primary care provider visits, emergency department visits, nonpsychiatric hospitalizations, and outpatient mental health visits. We determined the association between screening positive for the mental health conditions and health care utilization using both multivariable logistic regression and Poisson regression methods while controlling for sex, age, race, income, insurance status, marital status, educational level, and the presence of chronic medical conditions.

Results: After controlling for potential confounders, generalized anxiety disorder, panic disorder, and posttraumatic stress disorder were statistically significantly associated with more PCP visits, ED visits, and nonpsychiatric hospitalizations. Neither major nor minor depression were associated with more PCP visits, ED visits, or nonpsychiatric hospitalizations, except that minor depression was associated with $103 \%$ increase in PCP visits $(P<.001)$. Alcohol use disorder was associated with $16 \%$ fewer PCP visits $(P=.01)$ but $238 \%$ more nonpsychiatric hospitalizations $(P<.001)$.

Conclusions: After controlling for confounders we found that mental health conditions among a sample of family medicine patients were associated with increased use of ED services, nonpsychiatric hospitalizations, and, to a lesser extent, PCP visits. (J Am Board Fam Med 2008;21:398-407.)

Mental health conditions are common among adult primary care patients and contribute to lower functional status and increased health care utilization. ${ }^{1-3}$ In family medicine mental health conditions represent important risk factors for chronic medical illness, have implications for treatment decisions, and may affect outcomes of comorbid medical illness. ${ }^{4,5}$ However, these conditions often go

This article was externally peer reviewed.

Submitted 27 March 2007; revised 4 April 2008; accepted 8 April 2008.

From the Department of Family Medicine, University of Rochester, New York (CTF); the Department of Family Medicine, Boston University, Massachusetts (SS, VKC, LC).

Funding: This work was supported by the American Academy of Family Physicians Advanced Research Training Grant and unrestricted funds from Pfizer, Inc.

Conflict of interest: none declared.

Corresponding author: Colleen T. Fogarty, MD, MSc, University of Rochester, Highland Family Medicine, 777 South Clinton Avenue, Rochester, NY 14620 (E-mail: colleen_fogarty@urmc.rochester.edu). unrecognized and untreated by family physicians and other primary care clinicians. ${ }^{6-9}$

Research suggests that patients with mental health conditions use general medical services at a higher rate than those without mental health conditions. ${ }^{10,11}$ Seriously mentally ill individuals had higher rates of outpatient care when compared with the general population. ${ }^{12}$ Berren et $\mathrm{al}^{13}$ found overall lower health care costs but a much higher use of emergency department and lower use of outpatient medical care among severely mentally ill patients who were publicly insured. Conversely, providing outpatient mental health services to people with mental health conditions may reduce their overall general medical expenses compared with their untreated peers ${ }^{14,15}$ In one international study, comorbid medical conditions consistently increased health care costs and had greater influence on health care costs than depression alone. $^{16}$

Campbell et $\mathrm{al}^{17}$ reported decreased health expenditures and inpatient medical hospitalizations 
among patient panels of physicians who recorded more mental health diagnoses. Intervention by primary care providers with these patients can lead to lower health care utilization. ${ }^{3}$ Patients who frequently present to emergency departments were found to have higher rates of psychiatric, addiction, and social work services, as well as primary care and nonpsychiatric admissions. ${ }^{18}$ The availability of insurance also has been reported to significantly lower emergency department cost and utilization. ${ }^{19}$

Depression and anxiety are 3 times more prevalent among low-income groups as compared with high-income groups, ${ }^{20}$ and low income or uninsured status has been recognized as a barrier to care in the United States. ${ }^{21,22}$ Within Massachusetts, a state-funded "free care" program has provided full access to primary care and specialty ambulatory, emergency, and inpatient services for low-income and uninsured patients not eligible for Medicaid. The Boston HealthNet community health center network provides free health care to these individuals; this plan also includes pharmacy coverage. This state-funded program provides an environment with few financial barriers to care for an otherwise indigent population. We examined the impact of depression and anxiety on health care utilization within this population.

We conducted our study in 3 family medicine practices in the Boston HealthNet to better understand the health care utilization patterns of patients with mental health conditions. We hypothesized that patients with mental health conditions would demonstrate higher use of other medical services. The results of this study will inform clinical practices aimed at improving recognition, treatment, and management of individuals with mental health conditions.

\section{Methods}

\section{Setting}

We conducted our study in 3 urban family medicine practices affiliated with Boston University Medical Center and Boston HealthNet, including a hospital-based family medicine faculty practice and 2 community health center-based family medicine practices. These serve a predominantly low-income and ethnically diverse urban population.

\section{Participant Protections}

We obtained a Certificate of Confidentiality from the National Institute of Mental Health and ap- proval from the Boston University Medical Center Institutional Review Board. Participants read and initialed an informed consent form before enrolling in the study. We removed identifying information from the survey instrument and consent forms and, after data collection, destroyed the information linking participant name to study identification number.

\section{Participants}

A research assistant recruited a convenience sample of English-speaking adults. Between June 2001 and April 2002, a research assistant recruited participants from morning, afternoon, and evening clinical sessions of each of the family medicine clinicians (physicians and mid-level clinicians). To approximate a random sample, the research assistant approached every English-speaking patient at registration when there were few providers on staff during a session. During sessions with more providers, the research assistant approached approximately equal numbers of all providers' patients. Pregnant women and patients under the age of 18 were excluded from the study.

Participants completed the survey while waiting for their clinician, either in a private area of the reception area or in the examination room. The research assistant offered assistance to those with difficulty reading or understanding the questions. Five hundred sixty-two patients were approached and considered participating in the study. Of these, $510(91 \%)$ were eligible, $408(80 \%)$ consented to participate, and 367 completed the survey. These 367 participants represented a $72 \%$ response rate from among the eligible patients who were approached about participating. Patients who chose not to participate also did not consent for their medical or demographic data to be used in the study; therefore, we do not have comparison data between study participants and nonparticipants.

\section{Measures}

Demographics

Participants provided information regarding their sex; age; birthplace; race; income; insurance status (free care, Medicaid, other insurance, including Medicare); marital status; and educational level. The research assistant determined the presence of chronic medical conditions (hypertension, diabetes, back pain, arthritis, cardiac disease, asthma, chronic 
pain) through a medical record review, which was conducted after the patient visit.

\section{Mental Health Conditions}

To identify mental health conditions in this sample we used the Patient Health Questionnaire and used published scoring criteria. This screen is a primary care screening tool based on the Diagnostic and Statistical Manual of Mental Disorders, fourth edition (DSM-IV) containing items to screen for major depression, minor depression, generalized anxiety disorder (GAD), panic disorder (PD), and alcohol use disorder. ${ }^{23,24}$ To assess for posttraumatic stress disorder (PTSD) we used the short screening scale for DSM-IV PTSD, a 7-item, DSM-IV-based selfreport screening tool with reported sensitivity of $80 \%$ and specificity of $97 \% .^{25}$

\section{Health Care Utilization}

Our survey included measures of self-reported health utilization over the prior year for the following medical services: nonpsychiatric hospitalizations, emergency department visits, primary care provider visits, and mental health center visits.

\section{Data Management and Analysis}

We entered data with the Teleform program (Cardiff, Vista, CA) and imported it into SAS software (SAS Institute, Inc., Cary, NC) and S-PLUS 6 software (Insightful Corporation, Seattle, WA) for analysis. All analyses were done at $95 \% \mathrm{CI}$ and $\alpha=$ 0.05 levels. Because this was an exploratory study, we made no adjustment for multiple analyses.

After scoring the 6 mental health diagnoses by published scoring criteria, we dichotomized each variable (present or absent). We constructed composite variables for mental health conditions as follows: "any depressive diagnosis" consists of those who screened positive for major or minor depressive disorder; "any anxiety condition" consists of those who screened positive for GAD, PD, or PTSD (but not the full DSM list of anxiety disorders); and "any mental health disorder" includes all patients who screened positive for at least 1 of the 6 conditions measured. We examined differences in the distribution of each diagnosis based on age, race, educational attainment, income, and insurance status using $\chi^{2}$ anal- ysis for categorical variables and the Mantel Haenszel test of trend for ordinal variables (ie, educational level and income).

To assess the association between mental health conditions and medical utilization, we created binary variables of self-reported health care utilization over the prior year for the following medical services: primary care doctor visits ( 1 or 2 or more visits); emergency department visit (0 or 1 or more visit); nonpsychiatric hospitalizations (0 or 1 or more admission); and mental health center visits (0 or 1 or more visits). We then conducted bivariate analyses using $\chi^{2}$ statistics to assess for relationships between demographic variables (including chronic medical conditions) and each mental health diagnosis and each health care utilization binary variable.

We used multivariate logistic regression analysis to determine the association between mental health diagnosis and each health care service, adjusting for the potential confounding influence of age, race, educational level, income, insurance status, and chronic medical conditions on medical service utilization (36 models). Given the significant associations identified in the logistic regression model for mental health utilization, we performed a second set of regression models adjusted for this variable in the models for primary care, emergency department, and nonpsychiatric hospitalization utilization (27 models).

During visual inspection of the health care utilization data, we found skewed distributions of utilization, ie, there were very few patients who had very large numbers of primary care visits, emergency department visits, and nonpsychiatric hospitalizations. Because multivariate logistic regression requires the use of dichotomous outcome variables, we recognized that this analytic method would lose information inherent in our skewed distribution. Poisson distribution analysis is well suited for the skewed distribution of our outcome data; this method uses continuous variables for the outcome, thus preserving the information in our data. The analytic principle underlying Poisson regression analysis assumes that the log of the occurrence rate is a linear function of the predictor variable in question. We constructed a Poisson regression model for each type of health care utilization using the number of visits as the continuous outcome variable. 


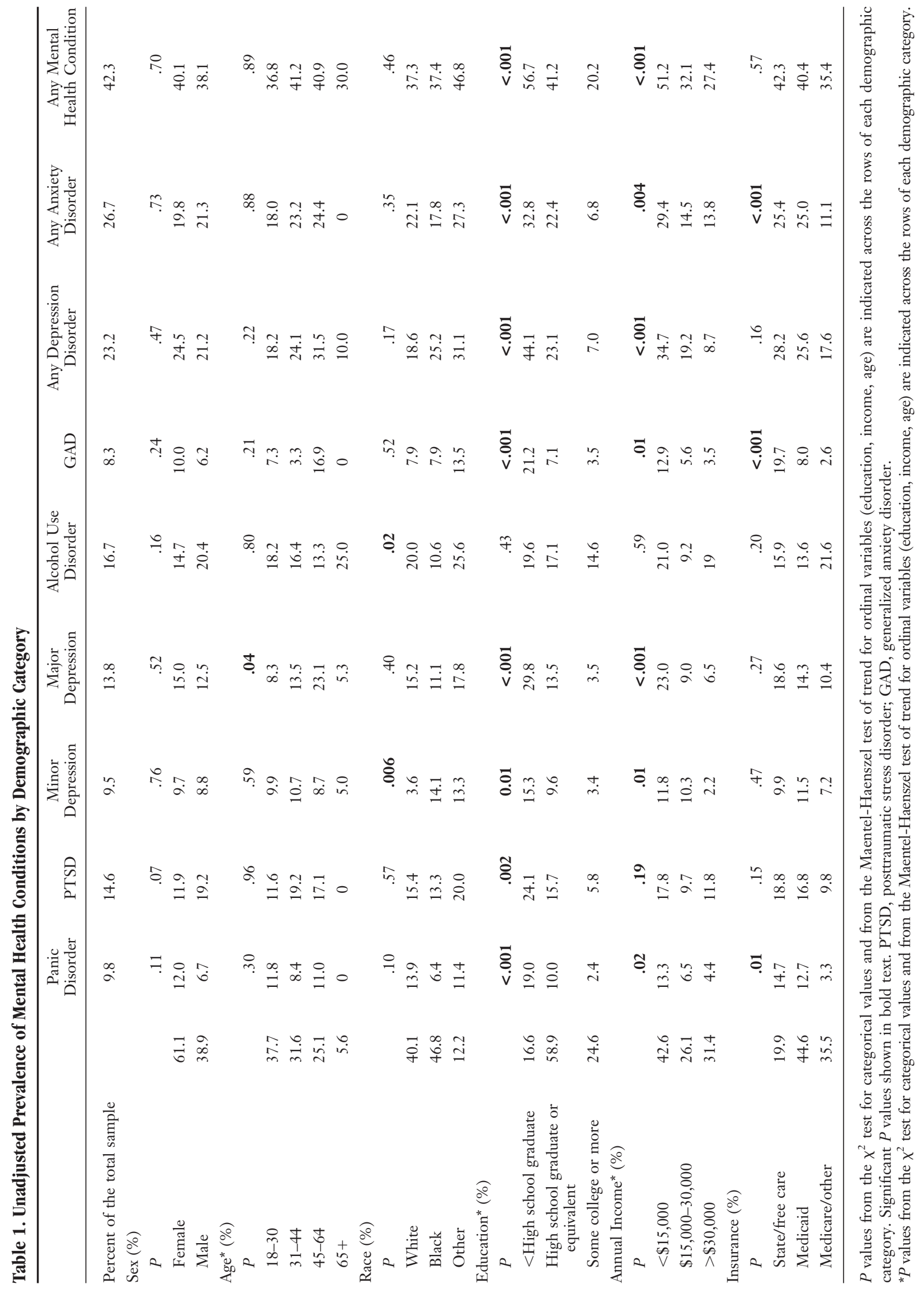


Table 2. The Association Between Mental Health Condition and Self-Reported Health Care Utilization With the Presence of a Chronic Medical Condition

\begin{tabular}{lcr}
\hline & Odds Ratio & $95 \%$ CI \\
\hline Mental Health Condition & & \\
Generalized anxiety disorder & 1.61 & $0.73-3.55$ \\
Panic disorder & 1.11 & $0.55-2.26$ \\
Posttraumatic stress disorder & 1.21 & $0.66-2.22$ \\
Minor depression & 1.69 & $0.83-3.44$ \\
Major depression & 2.53 & $1.35-4.71$ \\
Alcohol use disorder & 0.85 & $0.48-1.49$ \\
Any depression disorder & 2.34 & $1.42-3.87$ \\
Any anxiety disorder & 1.51 & $0.89-2.56$ \\
Any mental health condition & 1.69 & $1.10-2.58$ \\
Health Care Utilization (prior year) & & \\
PCP visit (2 or more visits vs 1 or none) & 1.29 & $0.83-1.99$ \\
ED visit (any visits vs zero) & 1.44 & $0.92-2.23$ \\
Nonpsychiatric hospitalization (any visits vs zero) & 1.75 & $0.97-3.15$ \\
MHC visit (any visits vs zero) & 0.76 & $0.39-1.46$ \\
\hline
\end{tabular}

PCP, primary care provider; ED, emergency department; MHC, mental health center. Bolded values are statistically significant.

\section{Results}

\section{Sample Demographics}

Of the 370 participants, $61.1 \%$ were women; $68.8 \%$ were aged 18 to 44 years; $83.4 \%$ had a high school education; and $42.6 \%$ had a household income less than $\$ 15,000$ per year. Almost half the sample $(46.8 \%)$ was black (observer coded) and the majority were single $(67.8 \%)$.

\section{Prevalence of Mental Health Conditions}

Mental health conditions were common in our patient population. The following prevalence of individuals screened positive: major depression, $13.8 \%$; minor depression, 9.5\%; GAD, 8.3\%; PD, 9.8\%; PTSD, 14.6\%; and alcohol use disorder, $16.7 \%$. Overall, $23.2 \%$ of respondents $(n=83)$ met criteria for any depressive disorder; $26.7 \%$ of respondents ( $\mathrm{n}=93$ ) met criteria for "any anxiety condition," and $42.3 \%$ of respondents $(n=155)$ met criteria for "any mental health disorder."

\section{The Association Between Mental Health and Chronic Medical Conditions with Demographic Variables}

Table 1 displays the associations between the demographic variables and the mental health conditions. Both educational level and income were significantly associated with all of the mental health conditions except alcohol use disorder.

Table 2 displays the association between mental health conditions and health care utilization given a chronic medical condition. Chronic medical conditions were associated with higher odds of each mental health disorder except alcohol use disorder. However, the increased odds were statistically significant only for major depression, "any depressive disorder," and "any mental health disorder." In this sample the presence of a chronic medical condition was not associated with higher health care utilization in any of the sectors studied.

\section{The Association Between Health Care Utilization and Demographics}

Table 3 shows the association between health care utilization and demographic variables. Lower educational attainment was associated with associated with Emergency department visits, nonpsychiatric hospitalizations, and mental health center visits. Race was associated with emergency department visits and primary care provider visits. Lower income and insurance status were associated with emergency department visits; approximately half of free care and Medicaid product participants visited the emergency department at least once compared with only a third of other insurance participants.

\section{Multivariate Analyses}

Given the associations between our demographic variables and chronic medical conditions with both mental health diagnoses and health care utilization, we adjusted all subsequent analyses for these con- 
Table 3. Prevalence of Health Care Utilization by Demographic Category

\begin{tabular}{|c|c|c|c|c|}
\hline & PCP Visit & ED Visit & Nonpsychiatric Hospitalization & MHC Visit \\
\hline \multicolumn{5}{|l|}{ Sex } \\
\hline$P^{\dagger}$ & .88 & .77 & .22 & .26 \\
\hline Female & 65.7 & 43.1 & 21.3 & 16.7 \\
\hline Male & 65.0 & 44.8 & 15.6 & 12.0 \\
\hline \multicolumn{5}{|l|}{ Age } \\
\hline$P^{*}$ & .22 & .78 & .09 & .63 \\
\hline $18-30$ & 61.0 & 43.6 & 14.7 & 13.9 \\
\hline $31-44$ & 67 & 43.1 & 16.7 & 14.6 \\
\hline $45-64$ & 66 & 49.4 & 27.0 & 17.6 \\
\hline $65+$ & 75 & 23.5 & 18.8 & 5.6 \\
\hline \multicolumn{5}{|l|}{ Race } \\
\hline$P^{\dagger}$ & .002 & .02 & .46 & .26 \\
\hline White & 70.6 & 36.6 & 21.9 & 18.6 \\
\hline Black & 66.5 & 52.8 & 15.9 & 11.8 \\
\hline Other & 42.6 & 40.5 & 17.1 & 11.8 \\
\hline \multicolumn{5}{|l|}{ Education } \\
\hline$P^{*}$ & .13 & $<.001$ & $<.001$ & .003 \\
\hline$<$ High school graduate & 75.0 & 56.0 & 29.8 & 33.3 \\
\hline High school graduate or equivalent & 62.9 & 48.4 & 21.1 & 10.4 \\
\hline Some college & 61.8 & 25.9 & 7.5 & 11.8 \\
\hline \multicolumn{5}{|l|}{ Annual Income } \\
\hline$P^{*}$ & .46 & .02 & .43 & .57 \\
\hline$<\$ 15,000$ & 63.6 & 46.4 & 17.8 & 16.4 \\
\hline$\$ 15,000-\$ 30,000$ & 64.6 & 45.1 & 27.7 & 10.6 \\
\hline$>\$ 30,000$ & 68.4 & 30.4 & 12.8 & 13.8 \\
\hline \multicolumn{5}{|l|}{ Insurance } \\
\hline$P^{\dagger}$ & .69 & .02 & .38 & .71 \\
\hline State/free care & 61.1 & 54.7 & 23.3 & 13.6 \\
\hline Medicaid & 65.2 & 47.8 & 19.7 & 16.7 \\
\hline Medicare/other & 67.2 & 34.2 & 15.0 & 13.2 \\
\hline
\end{tabular}

$P$ values are indicated across the rows of each demographic category; significant $P$ values are indicated in bold.

${ }^{*} P$ values from the Maentel-Haenszel test of trend for ordinal variables (education, income, age).

${ }^{\dagger} P$ values from the $\chi^{2}$ test for categorical values.

PCP, primary care provider; ED, emergency department; MHC, mental health center.

founding variables. The resultant logistic regressions of the relationship between mental health conditions and emergency department visits demonstrated increased odds of emergency department visits among those with PTSD, alcohol use disorder, and "any anxiety condition" (Table 4). Adjusted multivariable regression found that "any depression disorder" was associated with increased odds of primary care provider visits. All of the mental health conditions measured, with the exception of minor depression, were associated with significantly increased odds of mental health visits (Table 4).

Given the increased odds of mental health visits and the literature's suggestion that increased men- tal health visits might affect other health care utilization, we constructed a second set of logistic regression models for each mental health condition with each biomedical utilization variable (emergency department visit, primary care provider visit, and nonpsychiatric hospitalization). We adjusted for the potential confounding effects of demographics, chronic medical conditions, and mental health visits. After adjusting for mental health utilization, there remained only a few significant relationships (Table 5). "Any mental health disorder" resulted in double the odds of nonpsychiatric hospitalizations, whereas PTSD, "any mental health disorder," alcohol use disorder, and "any anxiety condition" were associated with approximately 
Table 4. Statistically Significant Associations Between Mental Health Conditions and Self-Reported Health Care Utilization in Prior Year, Adjusted for Demographic Variables and Chronic Conditions

\begin{tabular}{lccc}
\hline & Estimate & Odds Ratio & $95 \%$ CI \\
\hline PCP visits (1 vs 2 or more) & & & $1.09-4.39$ \\
$\quad$ Any depression disorder & 0.78 & 2.19 & $1.20-5.40$ \\
ED visits (zero vs any) & & & $1.08-4.18$ \\
$\quad$ Posttraumatic stress disorder & 0.93 & 2.54 & $1.09-3.96$ \\
Alcohol use disorder & 0.75 & 2.12 & \\
Any anxiety disorder & 0.73 & 2.08 & $1.26-11.18$ \\
MHC visits (zero vs any) & & & $1.87-15.27$ \\
Generalized anxiety disorder & 1.32 & 3.75 & $1.43-8.34$ \\
Panic disorder & 1.68 & 5.34 & $1.63-9.99$ \\
Posttraumatic stress disorder & 1.24 & 3.46 & $1.18-6.36$ \\
Major depression & 1.39 & 4.03 & $2.02-11.21$ \\
Alcohol use disorder & 1.01 & 2.74 & $1.89-10.01$ \\
Any depression disorder & 1.56 & 4.76 & $1.54-7.47$ \\
Any anxiety disorder & 1.47 & 4.34 & 3.39 \\
Any mental health condition & 1.22 & & \\
\hline
\end{tabular}

PCP, primary care provider; ED, emergency department; MHC, mental health center.

double the odds of reporting more than one emergency department visit over the previous year ( $\mathrm{Ta}-$ ble 5).

Poisson regression analyses identified changes in the percentage of visits in the presence of a given predictor variable. Table 6 shows the mental health conditions found to be significantly associated with health care utilization. All 3 anxiety disorders (GAD, PD, and PTSD) were associated with statistically significantly more primary care provider and emergency department visits and nonpsychiatric hospitalizations. Neither major nor minor depression was associated with more primary care provider visits, emergency department visits, or nonpsychiatric hospitalizations, except that minor depression was associated with $103 \%$ increase in primary care provider visits. Alcohol use disorder was associated with $16 \%$ fewer primary care provider visits but $238 \%$ more nonpsychiatric hospitalizations. All mental health conditions measured, except minor depression and PD, were associated with many times more self-reported mental health visits than among those respondents who did not meet criteria for mental health conditions.

\section{Discussion}

In a population among whom lack of insurance is not a direct financial barrier to obtaining care, all of the mental health disorders we measured were associated with increases in primary care, emergency department, and mental health clinic visits and

Table 5. Statistically Significant Associations Between Mental Health Conditions and Self-Reported Health Care Utilization in Prior Year, Adjusted for Demographic Variables, Chronic Conditions, and Mental Health Center Visits

\begin{tabular}{lccr}
\hline & Estimate & Odds Ratio & $95 \%$ CI \\
\hline $\begin{array}{l}\text { Hospitalization (zero vs any) } \\
\quad \text { Any mental health condition }\end{array}$ & 0.93 & & 2.54 \\
ED visits (zero vs any) & & & $1.14-5.63$ \\
Posttraumatic stress disorder & 1.01 & 2.74 & $1.26-5.95$ \\
Any mental health condition & 0.75 & 2.11 & $1.18-3.80$ \\
Alcohol use disorder & 0.91 & 2.48 & $1.18-5.19$ \\
Any anxiety disorder & 0.86 & 2.37 & $1.19-4.72$ \\
\hline
\end{tabular}

ED, emergency department. 
Table 6. Change in Self-Reported Health Care Utilization Associated with Mental Health Conditions, Adjusted for Demographic Variables, Chronic Conditions, and Mental Health Center Visits by Poisson Regression Analysis

\begin{tabular}{|c|c|c|c|c|c|c|c|c|}
\hline & \multicolumn{2}{|l|}{ PCP Visit } & \multicolumn{2}{|l|}{ ED Visit } & \multicolumn{2}{|c|}{$\begin{array}{l}\text { Nonpsychaitric } \\
\text { Hospitalization }\end{array}$} & \multicolumn{2}{|l|}{ MHC Visit } \\
\hline & $\begin{array}{c}\text { Change in visits } \\
(\%)\end{array}$ & $P$ & $\begin{array}{l}\text { Change in visits } \\
(\%)\end{array}$ & $P$ & $\begin{array}{l}\text { Change in visits } \\
(\%)\end{array}$ & $P$ & $\begin{array}{l}\text { Change in visits } \\
(\%)\end{array}$ & $P$ \\
\hline Generalized anxiety disorder & 28 & .002 & 122 & $<.001$ & 235 & $<.001$ & 523 & $<.001$ \\
\hline Panic disorder & 23 & .01 & 69 & .003 & 343 & $<.001$ & 13 & .71 \\
\hline PTSD & 32 & $<.001$ & 72 & $<.001$ & 148 & $<.001$ & 492 & $<.001$ \\
\hline Minor depression & 103 & $<.001$ & 20 & .17 & 23 & .69 & 29 & .11 \\
\hline Major depression & 7 & .16 & 28 & .07 & 7 & .61 & 575 & $<.001$ \\
\hline Alcohol use disorder & -16 & .01 & 33 & .03 & 238 & $<.001$ & 148 & $<.001$ \\
\hline Any depression disorder & 58 & $<.001$ & 30 & .03 & 13 & .7 & 487 & $<.001$ \\
\hline Any anxiety disorder & 9 & .1 & 71 & $<.001$ & 203 & $<.001$ & 357 & $<.001$ \\
\hline Any mental health condition & 30 & $<.001$ & 8 & .11 & 59 & .02 & 225 & $<.001$ \\
\hline
\end{tabular}

PCP, primary care provider; ED, emergency department; MHC, mental health center; PTSD, posttraumatic stress disorder. Bolded values are statistically significant.

nonpsychiatric hospitalizations. The presence of most of the mental health conditions (except alcohol use disorder) was associated with some increase in primary care visits, although not as dramatic an increase as observed with nonpsychiatric hospitalizations. The prevalence of mental health conditions in this sample of urban family medicine patients is similar to that reported in previous studies, with the exception of alcohol use disorder, which is higher in our sample. ${ }^{1}$

The strengths of this study are the use of family medicine practices for data collection, the use of validated measures of common mental health conditions, the use of Poisson regression analysis to extract the most information from the skewed distribution of the utilization data, and the adjustment for demographic variables, including insurance status. Conducting the study in family medicine practices allowed us to examine the prevalence of mental health conditions in our own care population but it also introduced possible biases in our sample selection. The inclusion of only English-speaking patients who had already presented for care may have impacted the prevalence of mental health conditions we found. On the other hand, our prevalence of mental health conditions, with the exception of alcohol use disorder, was similar to that reported in another urban primary care practice, ${ }^{1}$ although our prevalence of anxiety disorders was higher than that found in other studies. ${ }^{8,9}$ A relatively small sample size contributes to the risk of not detecting significant relationships where such relationships exist (type I error). The use of selfreported data for health care utilization in the prior year is subject to recall bias, and respondents who are more chronically physically or mentally ill may have either over- or under-reported their health care utilization. In addition, given the length of our survey instrument and the time taken to complete it, we did not use memory probes or other recall tools to help participants with their recall of health utilization. ${ }^{26} \mathrm{We}$ assessed chronic medical conditions by chart review and did not include objective assessment of functional status.

This analysis did not directly link the mental health conditions measured with the patient's medical record. In light of research demonstrating the under-recognition and under-treatment of mental health disorders by primary care and family physicians, ${ }^{6-9}$ we suspect that some respondents who met screening criteria for mental health conditions may not have been diagnosed or treated by their physicians for these conditions. Our data demonstrates a significantly elevated risk of emergency department visits and nonpsychiatric hospitalizations and mildly elevated risk of primary care provider visits after controlling for sex, age, race, income, insurance status, marital status, educational level, and the presence of chronic medical conditions among respondents who met criteria for 6 common mental health conditions. As expected, the presence of each condition was also associated with increased risk of use of mental health services. These findings provide further evidence of the in- 
teraction between mental health and biomedical health care utilization. Although our study adjusted for the confounding effects of chronic medical conditions on health care utilization, we recognize that the impact of mental health conditions on chronic medical conditions is likely to be bidirectional. We believe this study demonstrates the importance of diagnosing and treating and/or referring mental health conditions in all sectors of health care, including primary care providers, emergency department, nonpsychiatric inpatient visits, and mental health clinics. Our findings also provide support for the concept of onsite mental health services within primary care providers, emergency department, and nonpsychiatric inpatient units.

\section{References}

1. Olfson M, Shea S, Feder A, et al. Prevalence of anxiety, depression, and substance use disorders in an urban general medicine practice. Arch Fam Med 2000;9:876-83.

2. Olfson M, Fireman B, Weissman MM, et al. Mental disorders and disability among patients in a primary care group practice. Am J Psychiatry 1997;154: 1734-40.

3. Reifler DR, Kessler HS, Bernhard EJ, Leon AC, Martin GJ. Impact of screening for mental health concerns on health service utilization and functional status in primary care patients. Arch Intern Med 1996;156:2593-9.

4. Noel PH, Williams JW Jr, Unutzer J, et al. Depression and comorbid illness in elderly primary care patients: impact on multiple domains of health status and well-being. Ann Fam Med 2004;2:555-62.

5. Stover E, Fenton W, Rosenfeld A, Insel TR. Depression and comorbid medical illness: the National Institute of Mental Health perspective. Biol Psychiatry 2003;54:184-6.

6. Wang PS, Lane M, Olfson M, Pincus HA, Wells KB, Kessler RC. Twelve-month use of mental health services in the United States: results from the $\mathrm{Na}$ tional Comorbidity Survey Replication. Arch Gen Psychiatry 2005;62:629-40.

7. Kessler RC, Berglund P, Demler O, et al. The epidemiology of major depressive disorder: results from the National Comorbidity Survey Replication (NCS-R). JAMA 2003;289:3095-105.

8. Wittchen HU, Kessler RC, Beesdo K, Krause P, Hofler M, Hoyer J. Generalized anxiety and depression in primary care: prevalence, recognition, and management. J Clin Psychiatry 2002;63(Suppl 8):24-34.

9. Kroenke K, Spitzer RL, Williams JBW, Monahan PO, Lowe B. Anxiety disorders in primary care: prevalence, impairment, comorbidity, and detection. Ann Intern Med 2007;146:317-25.
10. Salsberry PJ, Chipps E, Kennedy C. Use of general medical services among Medicaid patients with severe and persistent mentali. Psychiatr Serv 2005;56:458-62.

11. Thomas MR, Waxmonsky JA, Gabow PA, FlandersMcGinnis G, Socherman R, Rost K. Prevalence of psychiatric disorders and costs of care among adult enrollees in a Medicaid HMO. Psychiatr Serv 2005; 56:1394-401.

12. Dickerson FB, McNary SW, Brown CH, Kreyenbuhl J, Goldberg RW, Dixon LB. Somatic healthcare utilization among adults with serious mental illness who are receiving community psychiatric services. Med Care 2003;41:560-70.

13. Berren MR, Santiago JM, Zent MR, Carbone CP. Health care utilization by persons with severe and persistent mental illness. Psychiatr Serv 1999;50: 559-61.

14. Schlesinger HJ, Mumford E, Glass GV, Patrick C, Sharfstein S. Mental health treatment and medical care utilization in a fee-for-service system: outpatient mental health treatment following the onset of a chronic disease. Am J Public Health 1983;73:422-9.

15. Simpson JS, Carlson LE, Trew ME. Effect of group therapy for breast cancer on healthcare utilization. Cancer Pract 2001;9:19-26.

16. Chisholm D, Diehr P, Knapp M, Patrick D, Treglia M, Simon G. Depression status, medical comorbidity and resource costs. Evidence from an international study of major depression in primary care (LIDO). Br J Psychiatry 2003;183:121-31.

17. Campbell TL, Franks P, Fiscella K, et al. Do physicians who diagnose more mental health disorders generate lower health care costs? J Fam Pract 2000; 49:305-10.

18. Byrne M, Murphy AW, Plunkett PK, McGee HM, Murray A, Bury G. Frequent attenders to an emergency department: a study of primary health care use, medical profile, and psychosocial characteristics. Ann Emerg Med 2003;41:309-18.

19. Davidson RA, Giancola A, Gast A, Ho J, Waddell R. Evaluation of access, a primary care program for indigent patients: inpatient and emergency room utilization. J Community Health 2003;28:59-64.

20. Sturm R, Gresenz CR. Relations of income inequality and family income to chronic medical conditions and mental health disorders: national survey. BMJ 2002;324:20-3.

21. Sareen J, Jagdeo A, Cox BJ, et al. Perceived barriers to mental health service utilization in the United States, Ontario, and The Netherlands. Psychiatr Serv 2007;58:357-64.

22. Vasiliadis H-M, Lesage A, Adair C, Wang PS, Kessler RC. Do Canada and the United States differ in prevalence of depression and utilization of services? Psychiatr Serv 2007;58:63-71. 
23. Spitzer RL, Kroenke K, Williams JB. Validation and utility of a self-report version of PRIME-MD: the PHQ primary care study. Primary Care Evaluation of Mental Disorders. Patient Health Questionnaire. JAMA 1999;282:1737-44.

24. Spitzer RL, Williams JB, Kroenke K, Hornyak R, McMurray J. Validity and utility of the PRIME-MD patient health questionnaire in assessment of 3000 obstetric-gynecologic patients: the PRIME-MD Patient Health Questionnaire Obstetrics-Gynecology Study. Am J Obstet Gynecol 2000;183:759-69.

25. Breslau N, Peterson EL, Kessler RC, Schultz LR. Short screening scale for DSM-IV posttraumatic stress disorder. Am J Psychiatry 1999;156:908-11.

26. Bhandari A, Wagner T. Self-reported utilization of health care services: improving measurement and accuracy. Med Care Res Rev 2006;63:217-35. 